\title{
Initial rupture of earthquakes in the 1995 Ridgecrest, California sequence
}

\author{
Jim Mori \\ U.S. Geological Survey, Pasadena CA 91106

\section{Hiroo Kanamori} \\ Seismological Laboratory, California Institute of Technology, Pasadena CA 91125
}

\begin{abstract}
Close examination of the $\mathrm{P}$ waves from earthquakes ranging in size across several orders of magnitude shows that the shape of the initiation of the velocity waveforms is independent of the magnitude of the earthquake. A model in which earthquakes of all sizes have similar rupture initiation can explain the data. This suggests that it is difficult to estimate the eventual size of an earthquake from the initial portion of the waveform. Previously reported curvature seen in the beginning of some velocity waveforms can be largely explained as the effect of anelastic attenuation; thus there is little evidence for a departure from models of simple rupture initiation that grow dynamically from a small region. The results of this study indicate that any "precursory" radiation at seismic frequencies must emanate from a source region no larger than the equivalent of a M0.5 event (i.e. a characteristic length of $\sim 10 \mathrm{~m}$ ). The size of the nucleation region for magnitude 0 to 5 earthquakes thus is not resolvable with the standard seismic instrumentation deployed in California.
\end{abstract}

\section{Introduction}

Several recent papers have suggested that the rupture initiation of earthquakes scales with earthquake size, and that it might be possible to determine the eventual size of the earthquake from the beginning of the $\mathrm{P}$ waveform. Umeda [1990] correlates earthquake magnitude with the duration of a low amplitude phase before the large energy release. Ellsworth and Beroza [1995] identify a nucleation phase of slow moment release and suggest that the duration of this feature scales with the eventual size of the earthquake. Iio [1995] has similar findings of slow beginnings for microearthquakes. Ishihara et al. [1992] present an opposing point of view in which larger earthquakes have faster initiation than smaller events. Other studies suggest an absence of scaling and conclude that the initiation of large earthquakes is similar to that of smaller events [Brune, 1979]. Abercrombie and Mori [1994] show that the beginning of the M7.3 Landers, California earthquake was similar to an M4.4 aftershock, and Anderson and Chen [1995] show that there were no systematic differences in the P-wave initiation of M3.0 - M8.0 events in Michoacan, Mexico. This study examines recordings of rupture initiation with a set of similarly recorded earthquakes that densely samples the magnitude range. We look for possible systematic variations in the waveforms that correlate with magnitude, but find no evidence for their existence.

Copyright 1996 by the American Geophysical Union.

Paper number 96GL02491

0094-8534/96/96GL-02491\$05.00

\section{Data}

The 1995 Ridgecrest sequence [Hauksson et al., 1996] provided closely spaced earthquakes over a range of magnitudes $\left(\mathrm{M}_{\mathrm{L}}\right.$ determined by the Southern California Seismic Network) that were recorded by nearby seismic stations of the USGS/Caltech Southern California Seismic Network. We examined $P$ waves from 49 earthquakes (M1.5 to M4.2) at hypocentral distances of 9 to $14 \mathrm{~km}$ recorded at station RGC (Fig. 1). The earthquakes were distributed in depth from 6 to 10 $\mathrm{km}$. Data were recorded on $1 \mathrm{~Hz}$ velocity sensors and digitized at 100 samples per second. The band-width of the system provides accurate recording of ground motion between 1 and 20 $\mathrm{Hz}$. This site had two channels running at different gain settings, so that there was a larger dynamic range than standard stations in the network. In order to obtain a representative sample, we used all the events of $M \geq 3.0$ well recorded at RGC from Sept. 26 to Oct. 31, 1995. A comparable number of smaller (M1.5 to 2.9) earthquakes, randomly chosen from a time period in early October, were added to the data set. Data were excluded only if noise levels obscured the beginning of the $\mathrm{P}$ wave. Such noise was caused primarily by codas of previous earthquakes. Because of the similarity in source-station geometry, direct comparisons can be made of the initiation of the $\mathrm{P}$ waveforms over the entire size range.

We also looked at the same earthquakes recorded on a more sensitive single-channel station CLC at hypocentral distances of 9 to $12 \mathrm{~km}$ (Fig. 1). This station clearly recorded smaller events down to M0.5 and was operating during larger M4 and M5 earthquakes that occurred prior to the installation of RGC on Sept. 26, resulting in a larger data set of 80 events. This station has limited dynamic range, but it provides a high magnification recording of the onsets for a wider magnitude spread of earthquakes.

The initial portions of the $P$ waves recorded at RGC and CLC are shown as a function of magnitude in Figs 2 and 3, respectively. The traces have been consistently lined up on the initial arrival times at $0.05 \mathrm{~s}$ using cross-correlations with a typical trace. Examination of the record section suggests there is an uncertainty of about one sample interval $(0.01 \mathrm{~s})$ in picking the initiation. The beginning portions of the velocity waveforms have varying amplitudes in the first several hundredths of a second with a general trend of increasing slope with increasing magnitude. This increase in slope with magnitude can largely be attributed to attenuation effects that reduce the amplitude of the high frequency components and therefore have a greater effect on the smaller earthquakes [Anderson, 1986]. Data from the high-gain station CLC go off-scale almost immediately after the first arrival, so we are looking at just the step response of the instrument. However, these data are still useful for searching for the existence of small motions before the impulsive arrival. 


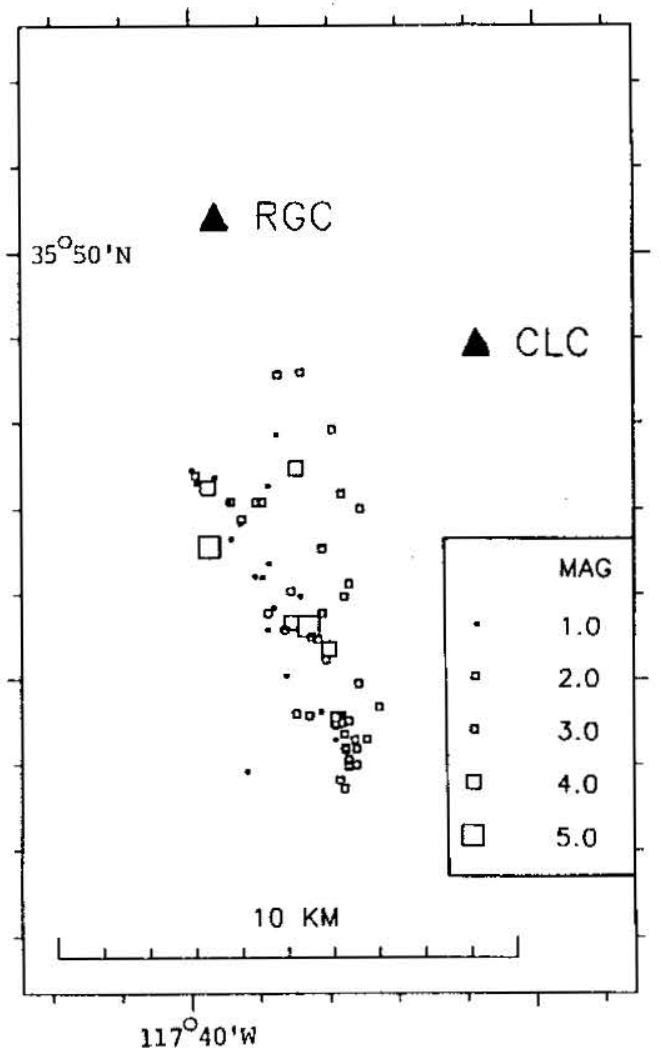

Figure 1. Locations of Ridgecrest events and stations of the Southern California Seismic Network used in this study.

\section{Estimate of Anelastic Attenuation}

An important part of this study is to show the effect that anelastic attenuation (Q) can have on the initial portion of the waveform. We estimated the whole-path attcnuation by comparing data to synthetic waveforms calculated for a range of $\mathrm{t}^{*}$, where $\mathrm{t}^{*}=$ travel time/Q .

For the data, we used a stacked waveform from RGC containing 9 events and from CLC using 11 events (Fig. 4). All the earthquakes in the stacks had magnitudes less than 1.0 , so that the source durations should be short compared to the combined attenuation and instrument operator. We used the stacked waveforms to average over the small variations in pulse width that are observed in the data (i.e., Fig. 2). There appears to be very little distance dependence of the pulse widths of the waveforms for these small events, suggesting that most of the attenuation occurs in the shallow region under the station. This allows us to use the same attenuation operator even though there are varying raypaths from the earthquakes.

The synthetics were calculated by convolving an attenuation operator [Carpenter, 1966] for various t* with the short-period instrument response [Stewart and O'Neill, 1980]. Fig. 4 shows synthetic waveforms for varying values of $\mathrm{t}^{*}$ along with the stacked waveforms from RGC and CLC. Both stations have a reasonable fit for $t^{*}=0.02 \mathrm{~s}$. A smaller value of $0.01 \mathrm{~s}$ can also fit the data given the variation in pulse widths, but values of $0.04 \mathrm{~s}$ and greater clearly produce waveforms that are too broad. In the modeling of the next section, we assume that $t^{*}=0.02 \mathrm{~s}$. Assuming a vaiue of $t^{*}=0.01 \mathrm{~s}$ would affect the absolute amplitudes, but not the overall pattern of the results.

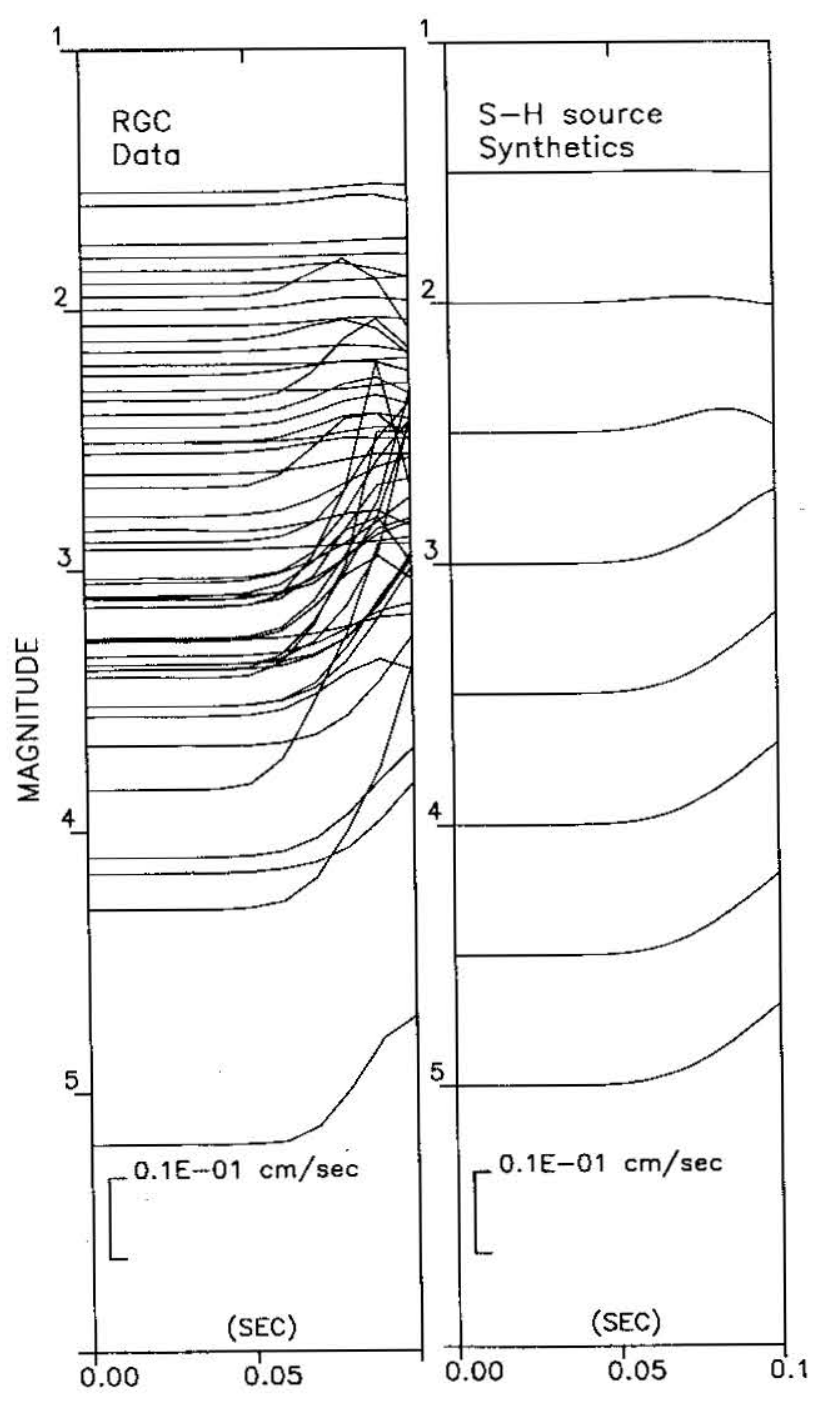

Figure 2. Beginning of $P$ waves recorded at RGC for M1.5 tc M4.2 earthquakes (left). Synthetic seismograms calculated with a Sato and Hirasawa source convolved with an attenuation operator using $t^{*}=0.02 \mathrm{~s}$ and the short-period instrument response (right).

\section{Synthetic Modeling}

We modeled the waveform initiation using a simple Sato and Hirasawa [1973] source. In this source model, the earthquake is a circular shear crack that expands radially at constant velocity, with all size earthquakes beginning in a similar manner. The differences for different size earthquake are controlled by the timing of the stopping of the rupture as it reaches its final radial dimension. Fig. 5 shows that this model predicts that the initiation of earthquakes would look the same regardless of their sizes. The Sato and Hirasawa source was calculated for a range of earthquake magnitudes corresponding to the Ridgecrest data. The shape of the waveforms are given by eqs 6 and 10 of Sato and Hirasawa [1973].

Computations were done using a stress drop of $3 \mathrm{MPa}$, a rupture velocity of $2.5 \mathrm{~km} / \mathrm{s}, \mathrm{P}$-wave velocity of $6 \mathrm{~km} / \mathrm{s}$, an $\mathrm{S}$ wave velocity of $3.5 \mathrm{~km} / \mathrm{s}$, a rigidity of $3.3 \times 10^{10} \mathrm{nts} / \mathrm{m}^{2}$, a density of $2.7 \mathrm{~g} / \mathrm{cm}^{3}$, distance $10 \mathrm{~km}$, and take-off angles $\theta=30^{\circ}$ and $\varphi=0^{\circ}$. We used a free surface amplification of 2.0 and a radiation pattern correction of 1.0. The source functions were 

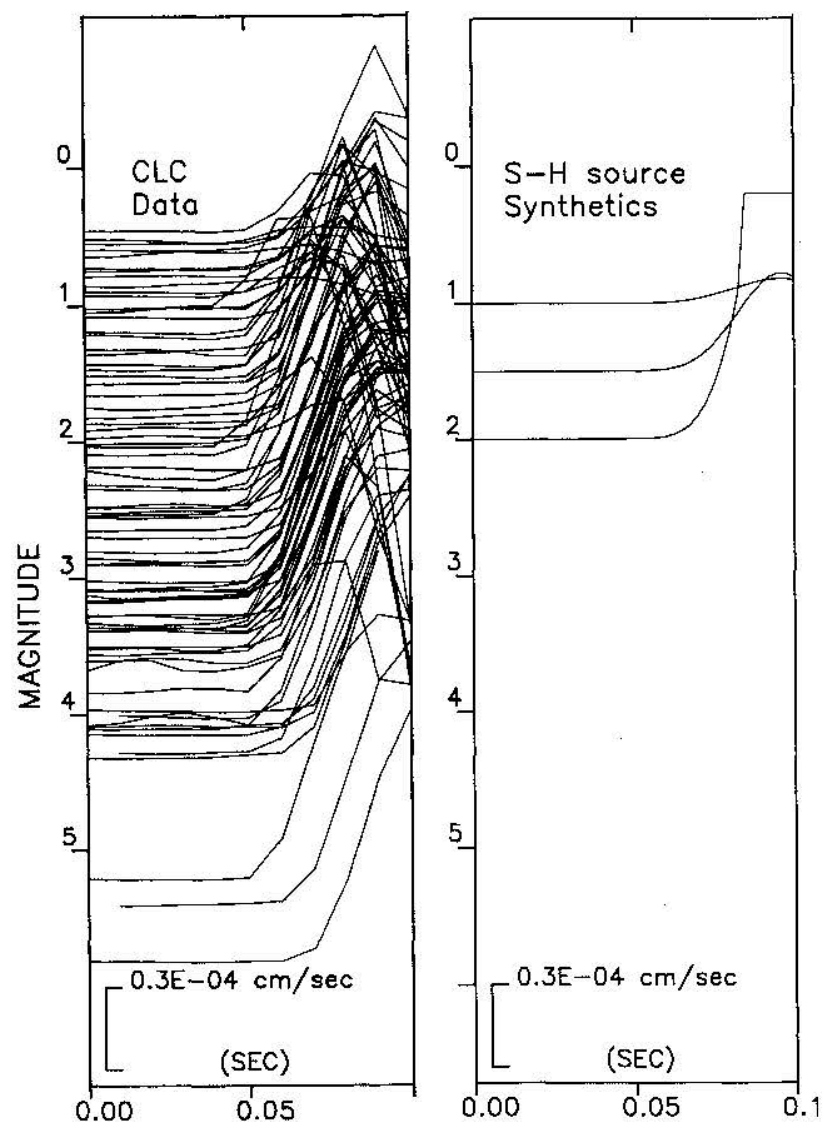

Figure 3. Beginning of $P$ waves recorded at CLC for M0.5 to 5.8 earthquakes (left). Synthetic seismograms calculated with a Sato and Hirasawa source convolved with an attenuation operator using $t^{*}=0.02 \mathrm{~s}$ and the short-period instrument response (right).

convolved with an attenuation operator using $\mathrm{t}^{*}=0.02 \mathrm{~s}$ and the instrument response. The synthetic waveforms are shown in the right-hand panels of Figs 2 and 3 . In order to make the amplitudes of the synthetic waveforms match the data, we arbitrarily set some of the amplitude factors, such as the radiation pattern and free-surface effect. Amplitude factors from 2 to 5 are commonly observed for different stations of the Southern California Seismic Network [Mori and Frankel, 1992].

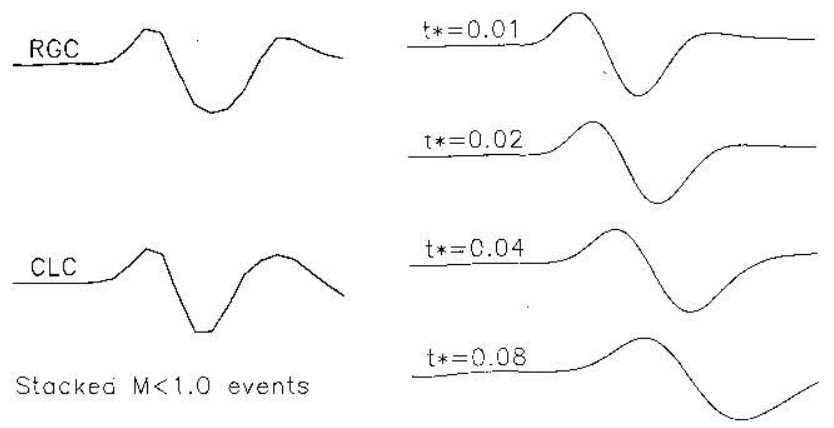

$0.00-\frac{(5 E C)}{0.10} \cdots \frac{1}{0.15}-\frac{1.20}{0.05}$

Figure 4. Stacked waveforms of small $(M<1.0)$ events recorded at RGC and CLC. Attenuation operators for various values of $t^{*}$ convolved with the short-period instrument response.

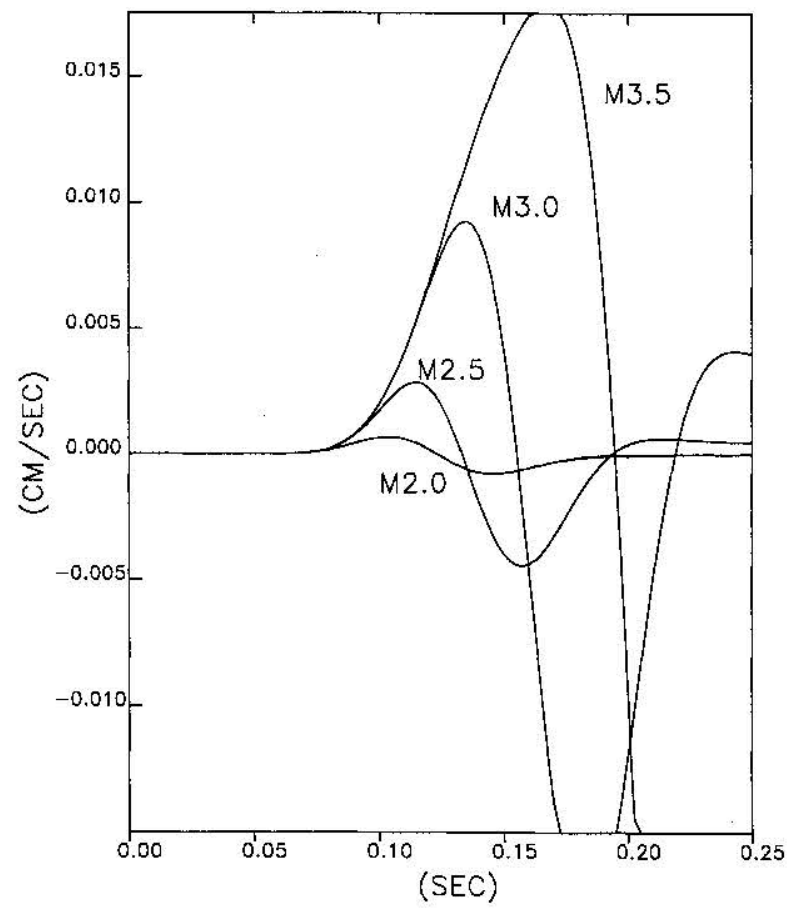

Figure 5. Initiation of P wave velocity waveforms of various magnitude for a Sato and Hirasawa model convolved with an attenuation operator using $t^{*}=0.02 \mathrm{~s}$ for a station $10 \mathrm{~km}$ from the source.

Even considering the amplitude adjustment described above, it is striking that the absolute amplitudes of the data are in general agreement with the synthetic waveforms over the whole magnitude range at $\mathrm{RGC}$. There are mismatches in the amplitudes by factors of about 2 or 3 between the synthetics and data, but we are able to generally match the slopes of the initiation for a range of over 3 magnitude units. For the smaller events ( $<\mathrm{M} 2.5)$, the source duration is less than $0.06 \mathrm{~s}$, which is shorter than the duration of the attenuation and instrument operator shown in Fig. 4. Therefore, at these smaller magnitudes, the waveforms reflect the attenuation and instrument response rather than the actual source duration. At CLC, the data go off-scale very quickly and there is little information about the amplitude of the arrival; however, we can see from these waveforms that there are no resolvable small motions before the $\mathrm{P}$ arrivals. These data thus place an upper bound on the size of any possible precursory motions. Since we can resolve the motion for M0.5 earthquakes and the P-wave onsets look similar for all size earthquakes from M0.5 to M5.4, this indicates that for the magnitude 4 and 5 earthquakes, any precursory arrivals, if they exist, must be smaller than the equivalent of a M0.5 event.

\section{Discussion}

The above detailed examination of the onset of a wide range of earthquakes did not show any strong magnitude dependence. There appear to be no systematic differences between initiation of small and large events. This is consistent with simple kinematic models such as those of Sato and Hirasawa [1973] and Boatwright [1980] that predict rupture initiation that is the same for all size earthquakes, assuming a constant stress drop [Kanamori and Anderson, 1975]. Fig. 5 shows the initiation of 
P-wave velocity waveforms from a Sato and Hirasawa source convolved with attenuation and instrument operators. In this simple model, earthquake rupture begins from a point and expands smoothly. The cventual size of the earthquake is determined by when the rupture is stopped. Our data from RGC are consistent with the absence of systematic differences of rupture initiation in the range from M1.5 to 4.3. The data from CLC cover an cven larger magnitude range, but are limited because the amplitudes saturate within a fow hundredths of a second. Despite the fact that the instrument is being driven out of its recording range, the clear onsets of the waveforms show that within the bandwidth of the instrument the initial ground motions are similar. That is, across this range of magnitudes (M0.5 to M5.8), there are no small amplitude $\left(10^{-7}\right.$ to $10^{-5}$ $\mathrm{m} / \mathrm{s}^{2}$ at 1 to $20 \mathrm{~Hz}$ ) precursors to the P-wave onset.

A further result of this study is that a simple source model, such as that of Sato and Hirasawa, convolved with reasonable attenuation operators can match the initiations of the observed waveforms. The Sato and Hirasawa models have a linear slope in the beginning of the velocity waveform. Assuming the values of t* estimated from recordings of small events, any observed "curvature" in the beginning of the waveform can be explained as the effect of anelastic attenuation. Thus, there is little evidence for significant rupture acceleration in the first few hundredths of a second. Instead, the data in this study indicate that ruptures grow dynamically very soon after the initiation. Any possible subcritical crack growth or "slow" beginnings, at frequencies in the seismic range, must occur within a few hundredths of a second and is not resolvable with current instrumentation in California.

We can use measurements of the velocity slope to estimate slip velocities and dynamic stress drop. In Fig 2 the slopes of the larger events (> M3.0) have values of $10^{-4}$ to $10^{-2} \mathrm{~m} / \mathrm{s}^{2}$ within $0.05 \mathrm{~s}$. Using the relationship for slip velocity and dynamic stress drop from eqs 10 and 41 from Boatwright [1980], gives slip velocities on the order of 0.003 to $0.3 \mathrm{~m} / \mathrm{s}$ and dynamic stress drops of 0.05 to $5 \mathrm{MPa}$.

Given the uncertainties due to radiation pattem and rupture velocity, the above values of slip velocities and dynamic stress drops are within the range of expected values for dynamic ruptures. We do not see any evidence in these data for magnitude-dependent slow rupture initiation, as suggested by Iio [1995] and Ellsworth and Beroza [1995], nor do we see any clear indications that larger earthquakes start faster as suggested by Ishihara et al. [1992].

\section{Conclusions}

Close examination of rupture initiation over a range of earthquake sizes does not show any systematic differences in time functions that depend on the eventual magnitude of the earthquake. The results of this study suggest that earthquakes of all sizes initiate in a similar manner and begin to grow dynamically within a few hundredths of a seconds of the rupture initiation. The final size of the event may be controlled more by the dynamic properties of the rupture and how the rupture is stopped, rather than by its initiation.
Acknowledgments. R. Geller, J. Gomberg, M. Kikuchi, M. Nakatani, and J. Vidale provided helpful suggestions on the manuscript. Data are available from the Southern California Earthquake Center Data Center. Contribution 5779, Division of Geological and Planetary Sciences, California Institute of Technology.

\section{References}

Abercrombie, R., and J. Mori, Local observations of the onset of a large earthquake: 28 June 1992 Landers, California, Bull. Seismol. Soc. Am. 84, 725-734, 1994.

Anderson, J., and Q. Chen, Beginnings of earthquake in the Mexican subduction zone on strong-motion accelerograms, Bull. Seismol. Soc. Am. 85, 1107-1116, 1995.

Anderson, J., Implications of attenuation for studies of earthquake source, in Das, S., J. Boatwright, C. Scholz, eds., Earthquake source mechanics, American Geophysical Union, Washington D.C., 311-318, 1986.

Boatwright, J., A spectral theory for circular seismic sources, simple estimates of source dimension, dynamic stress drop, and radiated seismic energy, Bull. Seismol. Soc. Am., 70, 1-29, 1980.

Brune, J,, Implications of earthquake triggering and rupture propagation for earthquake prediction based on premonitory phenomena, J. Geophys. Res., 84, 2195-2198.

Carpenter, E.W., Absorption of elastic waves - An operator for constant q mechanism, United Kingdom Atomic Energy Authority, Atomic Weapons Research Establishment Report D-43/60, 1966.

Ellsworth, W.L., and G. C. Beroza, Seismic evidence for an earthquake nuclcation phase, Science, 268, 851-855., 1995.

Hauksson, E., K. Hutton, H. Kanamori, L. Jones, J. Mori, S. Hough, and G. Roquemore, Preliminary report on the 1995 Ridgecrest earthquake sequence in castern California, Seismol. Res. Lett., 66, $55-60,1996$.

Iio, Y., Observations of the slow initial phase generated by microearthquakes: Implications for earthquake nucleation and propagation, J. Geophys. Res., 100, 15,333-15,349, 1995.

Ishihara, Y., Y. Fukao, I. Yamada, and H. Aoki, The rising slope of moment rate functions: The 1989 earthquakes off east coast of Honsiu, Geophys. Res. Lett., 19, 873-876, 1992.

Kanamori, H., and D.L. Anderson, Theoretical basis of some empirical relations in seismology, Bull. Seismol. Soc. Am., 65, 1073-1095, 1975.

Mori, J., and A. Frankel, Correlation of P-wave amplitudes and travel time residuals for teleseisms recorded on the southern California seismic network, J. Geophys. Res., 97, 6661-6674, 1992.

Sato, T., and T. Hirasawa, Body wave spectra from propagating shear cracks, J. Phys. Earth, 21, 415-431, 1973.

Stewart, S,W., and M.E. O'Neill, Calculation of the frequency response of the U.S.G.S. telemetered short-period seismic system, U.S. Geol. Survey. Open File Rep., 80-143, 1980.

Umeda, Y., High-amplitude seismic waves radiated from the bright spot of an earthquake, Tectonophysics, 175, 81-92, 1990.

J. Mori, US Geolgoical Survey, 525 South Wilson Av., Pasadena CA 91106 USA (email: mori@bombay.gps.caltech.edu)

II. Kanamori, Seismological Laboratory, California Institute of Technology, Pasadena CA 91125 USA (email: hiroo@seismo.gps.caltech.edu)

Received: 27 May1996 Revised: 5 Aug. 1996 Accepted: 5 Aug. 1996. 